\title{
Risco de iniciação ao tabagismo com o uso de cigarros eletrônicos: revisão sistemática e meta-análise
}

\author{
Risk of initiation to smoking with the use of electronic cigarettes: \\ systematic review and meta-analysis
}

\author{
Laura Augusta Barufaldi (https://orcid.org/0000-0001-9040-4399) ${ }^{1}$ \\ Renata Leborato Guerra (https://orcid.org/0000-0002-7507-6435) ${ }^{1}$ \\ Rita de Cássia Ribeiro de Albuquerque (https://orcid.org/0000-0002-3174-3689) ${ }^{1}$ \\ Aline do Nascimento (https://orcid.org/0000-0001-8801-6012) ${ }^{1}$ \\ Raphael Duarte Chança (https://orcid.org/0000-0002-1023-245X) ${ }^{1}$ \\ Mirian Carvalho de Souza (https://orcid.org/0000-0001-7516-1974) ${ }^{2}$ \\ Liz Maria de Almeida (https://orcid.org/0000-0002-2359-0651) ${ }^{3}$
}

\footnotetext{
${ }^{1}$ Núcleo de Avaliação de Tecnologias em Saúde, Divisão de Pesquisa Populacional, Instituto Nacional de Câncer José Alencar Gomes da Silva (INCA). Praça Cruz Vermelha 23, Centro. 20230-130 Rio de Janeiro RJ Brasil. lauraabarufaldi@gmail.com ${ }^{2}$ Divisão de Pesquisa Populacional, INCA. Rio de Janeiro RJ Brasil.

${ }^{3}$ Coordenação de Prevenção e Vigilância, INCA. Rio de Janeiro RJ Brasil.
}

\begin{abstract}
This article aims to evaluate the association between the use of electronic cigarettes and initiation to smoking, through a systematic review with meta-analysis of longitudinal studies. A bibliographic search was performed on the MEDLINE, Embase, LILACS and PsycInfo databases. Reference selection, data extraction and risk of bias assessment of the studies were independently carried out in pairs, and the disagreements were discussed with a third researcher to reach a consensus. Meta-analysis was performed using the Mantel-Haenszel random effects model. Among the 25 studies included, 22 evaluated the outcome of conventional cigarette experimentation and nine assessed the outcome of current smoking (in the last 30 days). The meta-analysis showed that the use of electronic cigarettes increased the risk of conventional cigarette experimentation by almost three and a half times ( $R R=3.42 ; 95 \% C I$ 2.81-4.15), and by more than four times the risk of current smoking ( $R R=4.32$; 95\%CI 3.13-5.94). The risk of smoking initiation is significantly higher among electronic cigarette users. The marketing authorization of such devices may represent a threat to public health policies in Brazil.

Key words Electronic Nicotine Delivery Systems, Smoking habit, Smoking Habit Prevention, Systematic Review, Meta-analysis
\end{abstract}

Resumo O objetivo deste artigo é avaliar a associação entre uso de cigarros eletrônicos e iniciação ao tabagismo, por meio de uma revisão sistemática com meta-análise de estudos longitudinais. Busca bibliográfica foi realizada nas bases MEDLINE, Embase, LILACS e PsycInfo. As etapas de seleção de referências, extração dos dados e avaliação do risco de viés dos estudos foi realizada em dupla, de forma independente e as divergências discutidas com um terceiro pesquisador para obtenção de consenso. Meta-análise foi realizada por meio do modelo Mantel-Haenszel de efeitos aleatórios. Dentre os 25 estudos incluidos, 22 avaliaramo desfecho de experimentação de cigarro convencional e nove avaliaram o desfecho de tabagismo atual (nos últimos 30 dias). A meta-análise demonstrou que o uso de cigarro eletrônico aumentou em quase três vezes e meia o risco de experimentação de cigarro convencional ( $R R=3,42$; IC95\% 2,814,15) e em mais de quatro vezes o risco de tabagismo atual $(R R=4,32$; IC95\% 3,13-5,94). O risco de iniciação ao tabagismo é significativamente maior entre usuários de cigarro eletrônico. A liberação da comercialização desses dispositivos pode representar uma ameaça para as políticas de saúde pública no Brasil.

Palavras-chave Sistemas Eletrônicos de Liberação de Nicotina, Tabagismo, Prevenção do Hábito de Fumar, Revisão Sistemática, Meta-análise 


\section{Introdução}

Os dispositivos eletrônicos para fumar (DEFs), incluindo cigarros eletrônicos, são produtos operados por bateria e utilizados para inalação de um aerossol, que normalmente contém nicotina, aromatizantes, aditivos de sabor e outros produtos químicos. Sua aparência pode ser similar à de cigarros tradicionais, charutos ou cachimbos, sendo os mais modernos semelhantes a canetas ou pen drives ${ }^{1}$.

O uso de cigarros eletrônicos expõe o organismo a uma variedade de produtos químicos como aqueles adicionados, provenientes do próprio dispositivo (nanopartículas de metal) e, ain$\mathrm{da}$, gerados durante o processo de aquecimento ou vaporização. Alguns produtos contidos no vapor de cigarros eletrônicos incluem carcinógenos conhecidos e substâncias citotóxicas, potencialmente causadoras de doenças pulmonares e cardiovasculares ${ }^{2}$.

Além dos riscos relacionados ao uso desses dispositivos, um ponto preocupante em termos de saúde pública é a possibilidade de o cigarro eletrônico servir como um produto introdutório para outros produtos derivados de tabaco, especialmente entre populações mais vulneráveis, como adolescentes e adultos jovens ${ }^{3}$. De fato, alguns estudos longitudinais sugerem que o uso de cigarros eletrônicos está associado à iniciação do tabagismo com cigarro convencional entre jovens que nunca havia fumado ${ }^{4-7}$.

Duas revisões sistemáticas sobre cigarro eletrônico e iniciação do tabagismo foram publicadas anteriormente ${ }^{3,4}$. Soneji et al. ${ }^{3}$ conduziram uma revisão sistemática com meta-análise, publicada em 2017, que apontou para um risco aumentado de iniciação de cigarros convencionais associado ao uso de cigarro eletrônico. Outra revisão sistemática mais recente ${ }^{4}$ foi baseada em uma busca realizada somente em uma base de dados eletrônica (PubMed) e não apresentou uma medida sumário.

No Brasil, ações nacionais de controle do tabagismo implementadas ao longo dos últimos 30 anos têm apresentado êxito na redução de sua prevalência na população, inclusive entre adolescentes e adultos jovens. Segundo um estudo que comparou duas edições de um inquérito nacional sobre a saúde de escolares, houve uma queda na prevalência de uso atual de cigarros de 6,3\% em 2009 para 5,4\% em 2015 (variação média anual: $-0,2 \%)^{5}$. Entretanto, a frequência de experimentação de cigarros convencionais permanece expressiva nessa população. Entre alunos de 13 a 15 anos, a frequência foi de 19\%, chegando a pouco mais de $29 \%$ entre aqueles com idade de 16 e 17 anos. Neste grupo (16 a 17 anos), 10\% experimentou cigarros antes dos 14 anos de idade e aproximadamente $8 \%$ consumiram cigarros pelo menos uma vez nos últimos 30 dias anteriores à pesquisa ${ }^{6}$.

Apesar da comercialização, importação e propaganda de cigarros eletrônicos ser proibida no Brasil pela Agência Nacional de Vigilância Sanitária desde 2009 (RDC 46 28/08/2009), esses produtos são vendidos ilegalmente pela internet, pelo comércio informal ou, ainda, podem ser adquiridos no exterior para uso pessoal, de maneira que já existem evidências sobre sua utilização no Brasil ${ }^{7,8}$. A disseminação de cigarros eletrônicos no país tem enorme potencial para afetar as políticas públicas de saúde, considerando a sua possível relação com o aumento da iniciação ao tabagismo e a conhecida associação deste com o desenvolvimento de diferentes doenças e tipos de câncer.

Portanto, a atualização das revisões feitas anteriormente sobre esse tema é importante para monitorar esse cenário e para o planejamento de ações de prevenção e combate ao tabagismo. O objetivo deste estudo foi realizar revisão sistemática com meta-análise de estudos longitudinais que avaliaram a associação entre experimentação e uso habitual de cigarros eletrônicos e o consumo subsequente de cigarros convencionais.

\section{Métodos}

Esse estudo seguiu as recomendações do Preferred Reporting Items for Systematic Reviews and Meta-Analyses (PRISMA) 9 . A partir da pergunta de pesquisa foi estruturado o acrônimo PECOS: $\mathrm{P}$ (população): indivíduos não tabagistas; E (exposição): uso de dispositivos eletrônicos para fumar com nicotina; C (controle ou comparador): não uso de dispositivos eletrônicos para fumar com nicotina; $\mathrm{O}$ (outcome ou desfecho): iniciação ao tabagismo e S (study ou desenho de estudo): estudos longitudinais.

\section{Fontes de informação e buscas}

Inicialmente, uma busca por revisões sistemáticas, contemplando termos relacionados à exposição (dispositivos eletrônicos para fumar) foi realizada nas bases MEDLINE (via PubMed), Embase, Cochrane Library e BVS Regional/LILACS. Duas revisões sistemáticas sobre iniciação ao 
tabagismo foram, então, utilizadas como ponto de partida para uma busca mais específica ${ }^{3,4}$.

Nesta segunda busca, além dos termos relacionados à exposição (dispositivos eletrônicos para fumar), a estratégia incluiu termos relacionados ao desfecho (iniciação ao tabagismo). Um filtro de data (01/01/2018) foi introduzido a partir da data da busca da revisão sistemática de Glasser et al. ${ }^{4}$, (material suplementar). Esta busca foi realizada em 09 de janeiro de 2020 nas bases MEDLINE (via PubMed), Embase, BVS Regional/LILACS e PsycInfo. Também foram revisadas as listas de referências das revisões sistemáticas identificadas inicialmente e dos estudos selecionados.

\section{Critérios de elegibilidade}

Foram incluídos estudos que atenderam ao acrônimo PECOS, ou seja, estudos observacionais longitudinais que avaliaram a associação entre o uso de cigarro eletrônico e a iniciação ao uso de cigarro convencional entre não tabagistas. Não houve restrição relacionada ao idioma da publicação. Não foram incluídos outros desenhos de estudo, como artigos de opinião e editoriais, além das publicações que não disponibilizaram todos os dados necessários, como resumos de congresso.

\section{Seleção dos estudos}

A seleção foi feita por dois pesquisadores (RCRA e AN) de forma independente, primeiramente pela leitura de títulos e resumos e, em seguida, pela avaliação dos textos completos, utilizando o programa Rayyan ${ }^{10}$. As divergências foram discutidas com um terceiro pesquisador (LAB) até se chegar a um consenso.

\section{Extração dos dados}

A extração dos dados também foi realizada por dois pesquisadores de forma independente a partir deum formulário em planilha Microsoft Office Excel, previamente elaborado. As informações extraídas dos artigos selecionados incluíram autor e ano de publicação, sigla do estudo, localidade, ano da linha de base, descrição da população, idade, sexo, definição da exposição e do desfecho, tempo de seguimento, covariáveis, número total de participantes, número de casos nos grupos exposição e controle e, por fim, estimativa da medida de associação. Para um dos artigos em que dados importantes estavam ausentes, as in- formações foram obtidas através de contato com os autores.

\section{Avaliação do risco de viés dos estudos}

A ferramenta ROBINS-I (Risk Of Bias In Non -randomized Studies - of Interventions), na sua versão para estudos de coorte ${ }^{11}$, foi utilizada para avaliação do risco de viés dos estudos primários. Essa etapa também foi realizada de forma independente por uma dupla de pesquisadores e as divergências discutidas para obtenção de consenso.

\section{Síntese dos resultados}

Uma meta-análise, utilizando os dados brutos, foi realizada para calcular a estimativa sumária de risco com seu intervalo de confiança de 95\%, por meio do modelo de Mantel-Haenszel de efeitos aleatórios. Essa análise foi realizada para dois desfechos: experimentação de cigarro convencional (o indivíduo havia, no mínimo, experimentado cigarro no período de acompanhamento) e tabagismo atual (o indivíduo havia fumado nos últimos 30 dias). A magnitude da heterogeneidade entre os estudos incluídos na meta-análise foi avaliada pela estatística $\mathrm{I}^{2}$, um resultado superior a $50 \%$ indica presença de heterogeneidade ${ }^{12}$. Como os estudos incluídos eram heterogêneos foi utilizado o modelo Mantel-Haenszel de efeitos aleatórios.

Análises de subgrupos foram realizadas para verificar diferenças nas seguintes variáveis: idade dos participantes ( $<18$ anos; $\geq 18$ anos), tamanho da amostra $(<1.500 ; \geq 1.500)$ e tempo de seguimento ( $\leq 1$ ano, $>1$ ano). Além disso, uma meta-análise foi realizada diretamente com as medidas de associação relatadas nos estudos que foram ajustadas para possíveis fatores de confundimento da população.

O viés de publicação foi avaliado através do gráfico de funil e do teste de Egger. Todas as análises foram realizadas no programa Stata, versão 15.

\section{Resultados}

Foram identificados nas buscas 543 estudos e excluídas as duplicatas $(\mathrm{N}=115)$, resultaram em 428 referências que, adicionadas aos estudos primários incluídos nas duas revisões sistemáticas ${ }^{3,4}$, totalizaram 439. Após a leitura de título e resumo, 48 estudos foram selecionados para leitura de texto completo. Para compor a meta-análise, 
foram incluídos 25 estudos longitudinais, publicados entre os anos de 2015 e 2019, que avaliaram a iniciação do uso de cigarro convencional após a exposição a cigarros eletrônico em não fumantes (Figura 1).

Vinte e um dos trabalhos foram desenvolvidos em países europeus e nos Estados Unidos ${ }^{13-33}$, dois foram realizados no Canadáa ${ }^{34,35}$, um em Taiwan ${ }^{36}$ e outro no $\mathrm{México}^{37}$. O tempo de seguimento dos estudos variou entre 6 meses e 2 anos e $80 \%$ $(n=20)$ deles incluiu, adolescentes e estudantes do ensino médio. $\mathrm{O}$ desfecho foi considerado como, pelo menos, a experimentação de cigarro convencional ( 1 ou 2 tragos) em 22 estudos ${ }^{14-19,21-30,32-37}$ e o seu uso atual (nos últimos 30 dias) em 9 estudos ${ }^{13-15,20,25,30-32,37}$. O risco geral de viés avaliado pela ferramenta ROBINS-I foi moderado ou baixo para a maioria dos estudos (Quadro 1).

Para o desfecho de experimentação de cigarro convencional o risco relativo (RR) não ajustado variou de 1,26 a 8,45 , para aqueles indivíduos que haviam experimentado cigarros eletrônicos em comparação com aqueles que não haviam experimentado cigarros eletrônicos na linha de base. A meta-análise dos dados dos vinte dois estudos demonstrou que o uso de cigarro eletrônico aumentou em quase três vezes e meia o risco de experimentação de cigarro convencional ( $\mathrm{RR}=3,42 ; \mathrm{IC} 95 \%$ 2,81-4,15). A heterogeneidade entre os estudos foi elevada ( $\left.\mathrm{I}^{2}=95 \%\right)$ (Figura 2 ).

Para o desfecho de tabagismo atual (nos últimos 30 dias), os valores de RR dos estudos variaram de 2,04 a 11,88 . Nesse caso, na medida combinada dos nove estudos foi observado que o uso de cigarros eletrônicos aumentou em mais de quatro vezes o risco de posterior utilização de cigarro convencional ( $R R=4,32$; IC95\% 3,13$5,94)$. Para essa análise a heterogeneidade entre os estudos foi um pouco mais baixa, mas ainda significativa $\left(\mathrm{I}^{2}=73,6 \%\right)$ (Figura 2$)$.

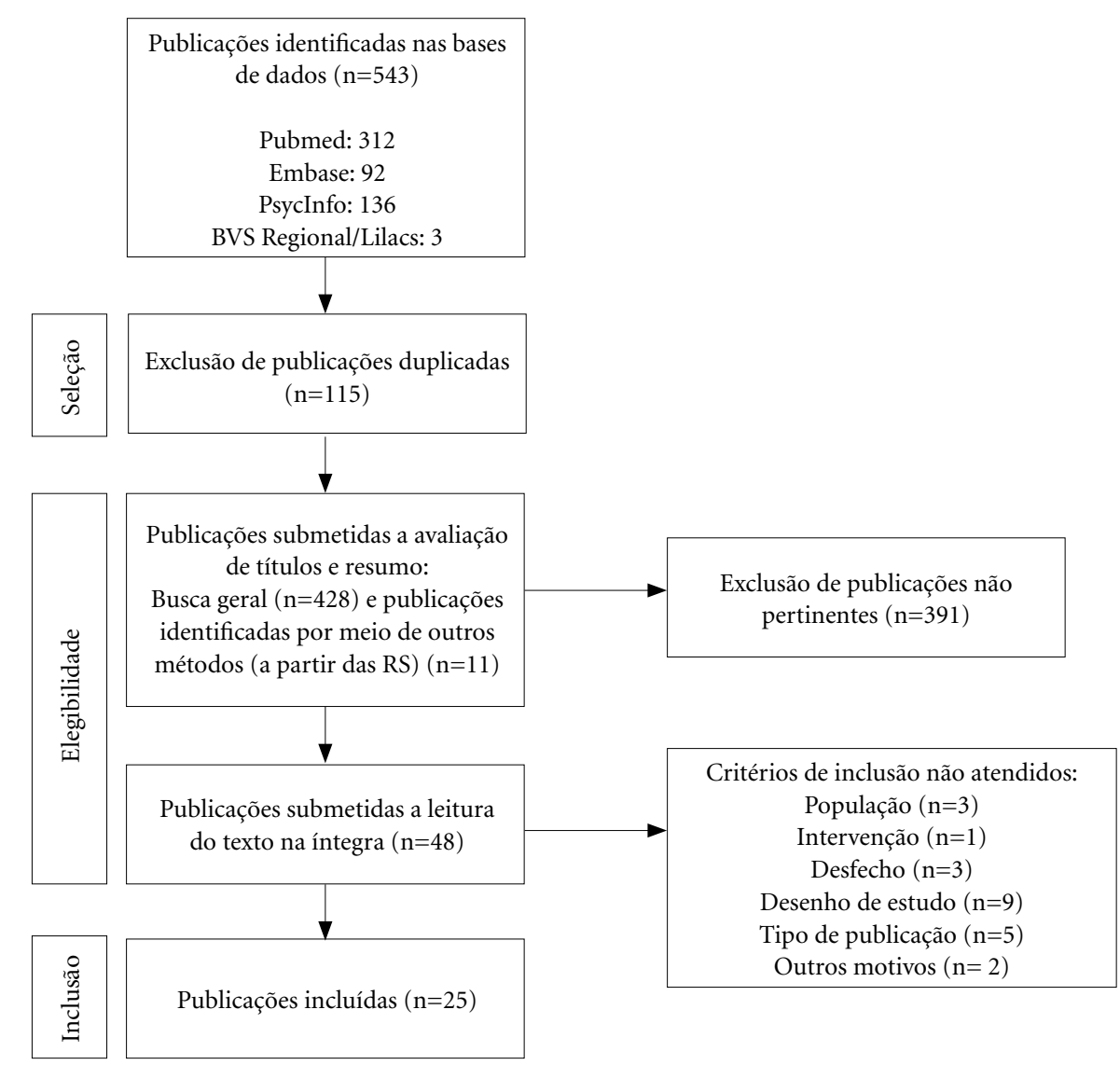

Figura 1. Fluxograma de seleção e elegibilidade dos estudos. 
6093

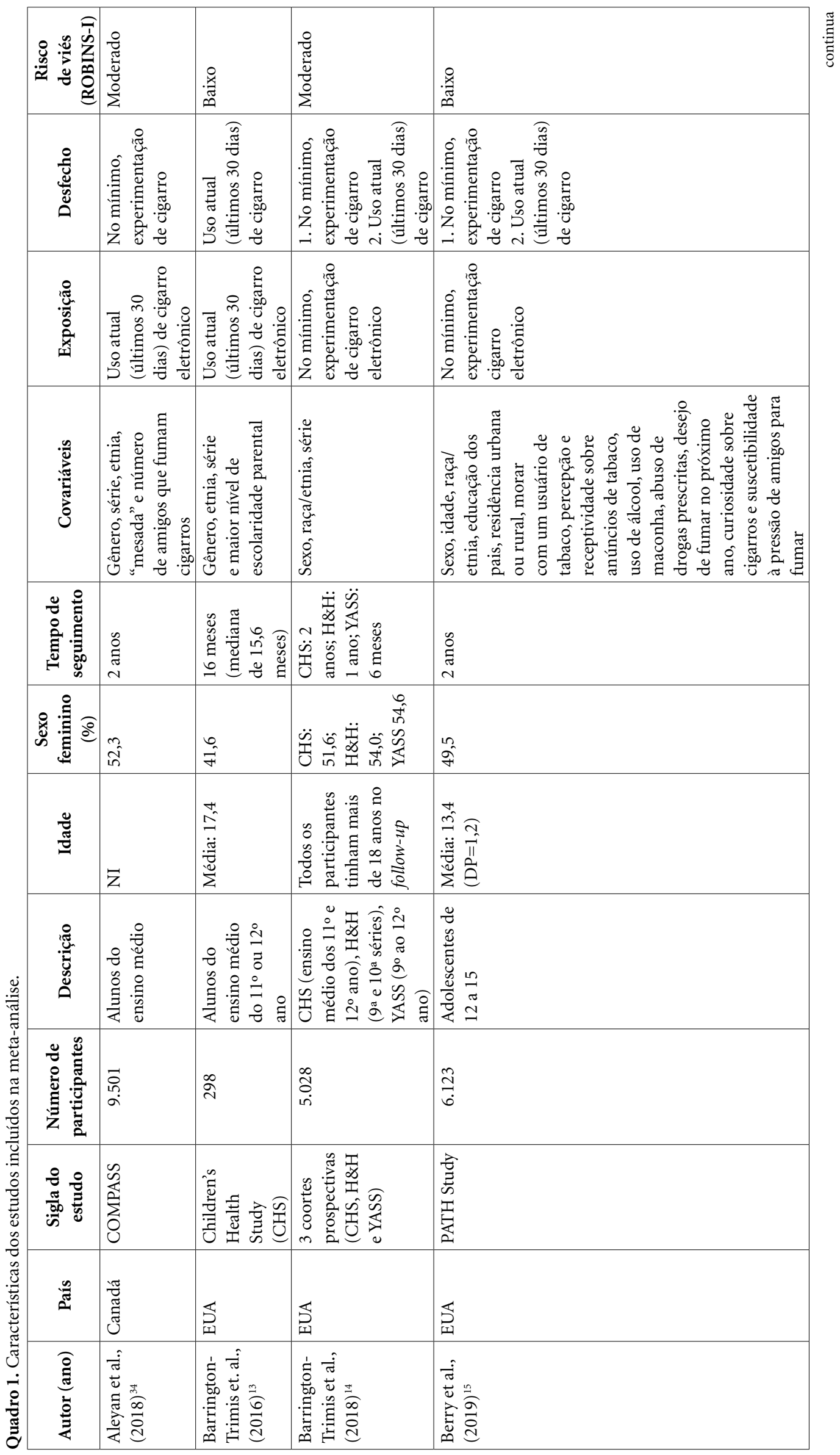




\begin{tabular}{|c|c|c|c|c|c|c|}
\hline 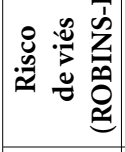 & . & . & 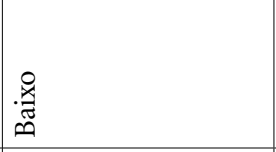 & 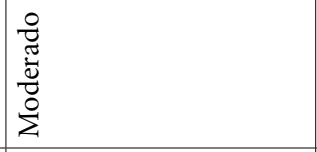 & 㫕 & 莺 \\
\hline 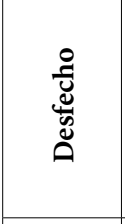 & 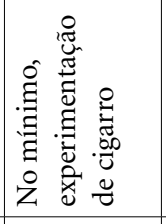 & 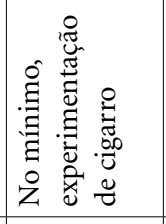 & 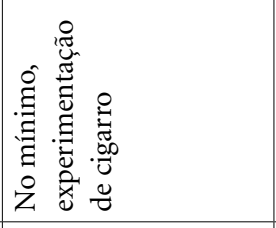 & 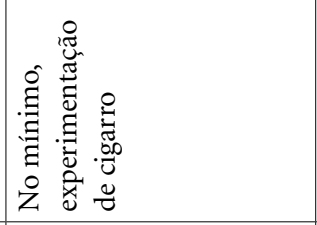 & 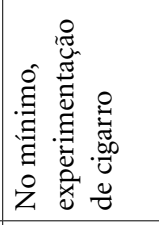 & 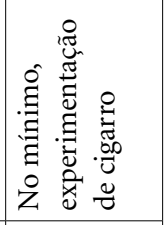 \\
\hline 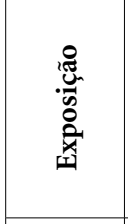 & 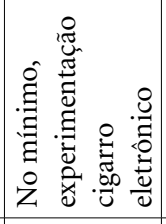 & 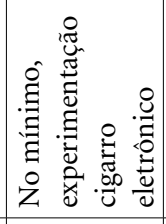 & 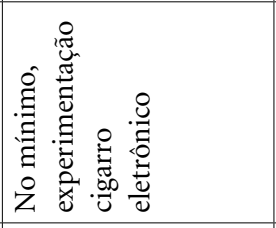 & 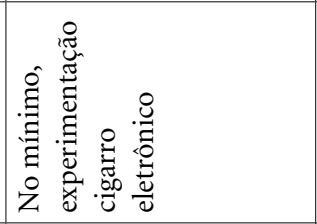 & 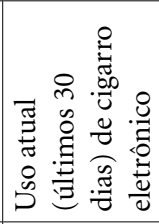 & 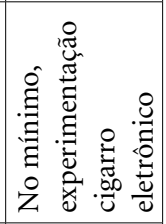 \\
\hline 这 & 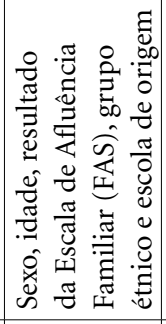 & 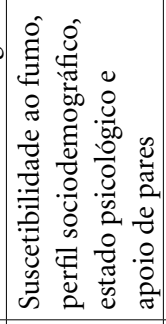 & 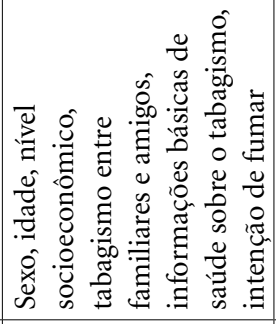 & 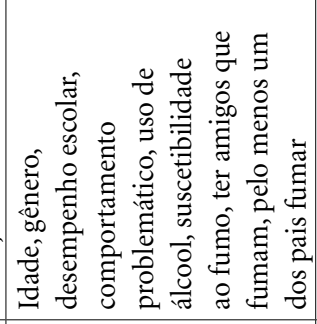 & 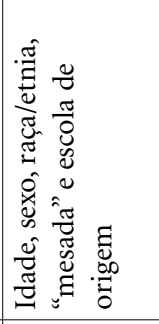 & 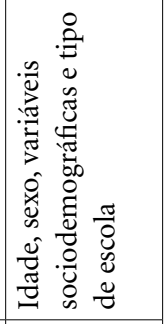 \\
\hline 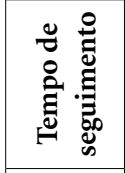 & ‡ & 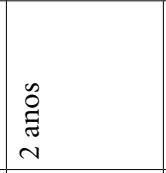 & 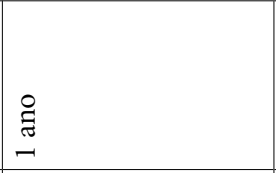 & 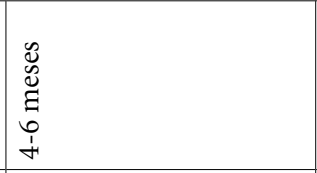 & 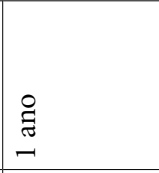 & 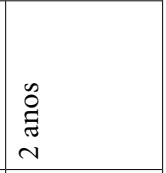 \\
\hline 咅 & Z & $\bar{z}$ & in & $\begin{array}{l}\infty \\
\hat{n} \\
\hat{n}\end{array}$ & $\stackrel{+}{\hat{n}}$ & $\begin{array}{l}0 \\
\dot{q}\end{array}$ \\
\hline 䞤 & 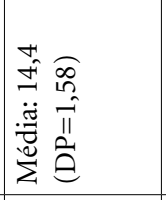 & 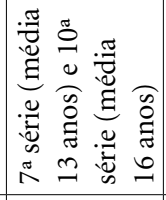 & 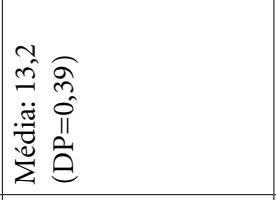 & $\begin{array}{l}0 \\
0 \\
\vdots \\
\sigma \\
\infty \\
0 \\
0 \\
= \\
=\end{array}$ & z & 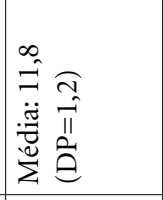 \\
\hline 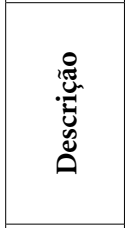 & 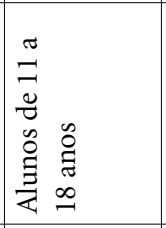 & 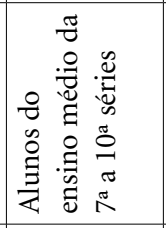 & 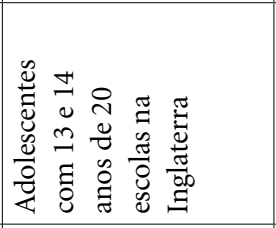 & 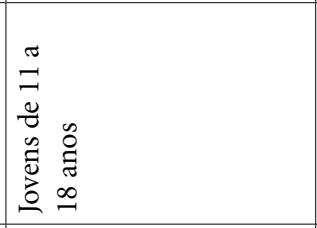 & 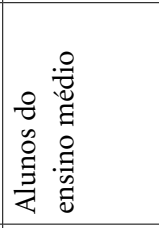 & 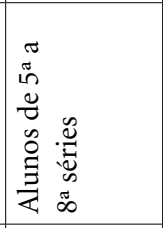 \\
\hline 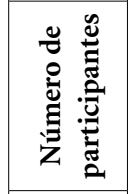 & $\underset{\stackrel{\sim}{\sim}}{\stackrel{i}{i}}$ & $\begin{array}{l}\stackrel{n}{\vec{\sigma}} \\
\stackrel{\Xi}{\exists}\end{array}$ & 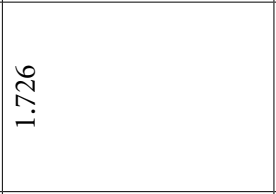 & $\tilde{\Omega}$ & 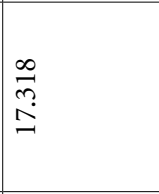 & $\begin{array}{l}\infty \\
\infty \\
\stackrel{\lambda}{n}\end{array}$ \\
\hline 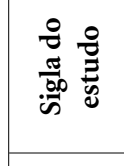 & 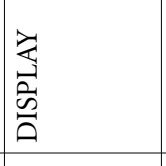 & 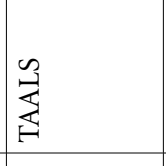 & $\bar{z}$ & 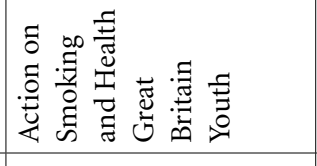 & $\begin{array}{l}n \\
\sum_{1} \\
0 \\
0 \\
0\end{array}$ & 竞 \\
\hline$\stackrel{\infty}{\tilde{E}}$ & 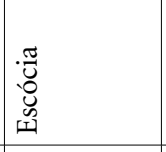 & 莺 & 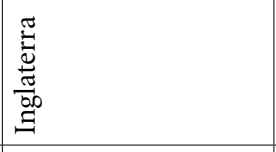 & 吾 & 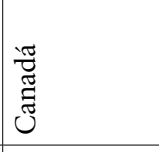 & 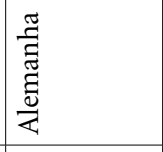 \\
\hline 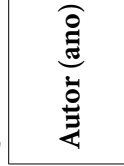 & 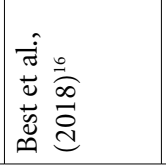 & 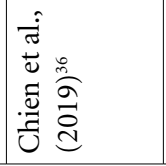 & 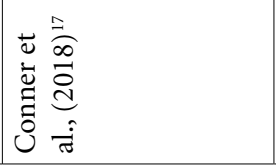 & 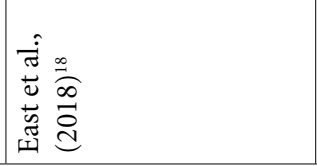 & 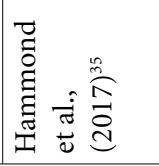 & 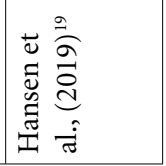 \\
\hline
\end{tabular}




\begin{tabular}{|c|c|c|c|c|c|c|}
\hline 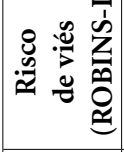 & 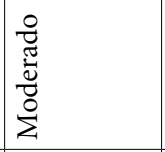 & 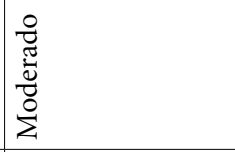 & 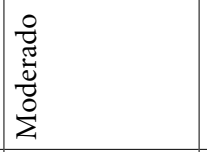 & 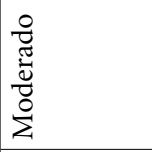 & 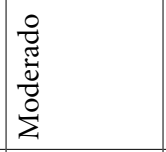 & 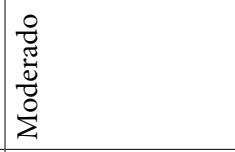 \\
\hline 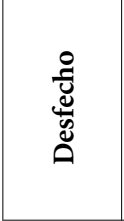 & 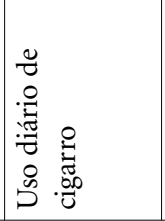 & 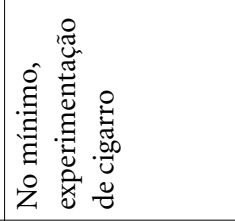 & 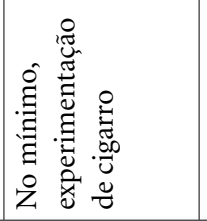 & 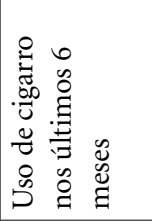 & 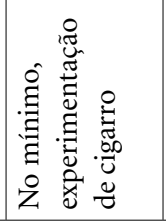 & 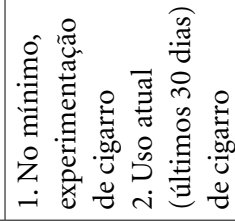 \\
\hline 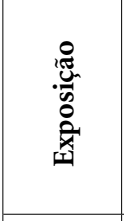 & 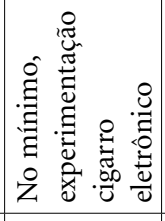 & 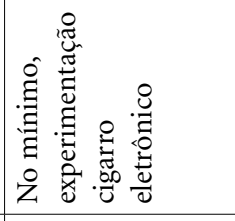 & 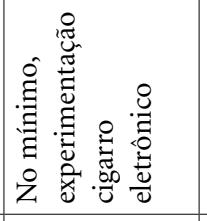 & 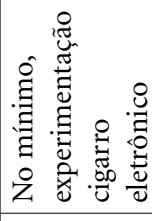 & 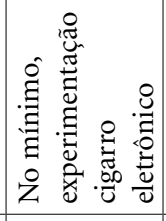 & 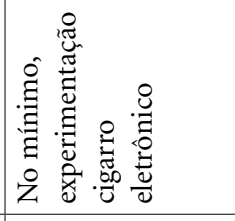 \\
\hline 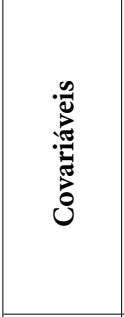 & 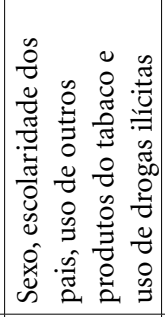 & 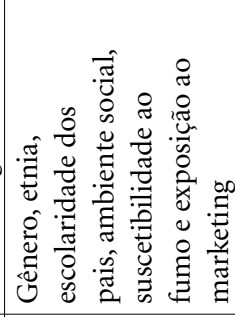 & 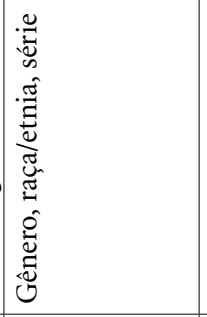 & 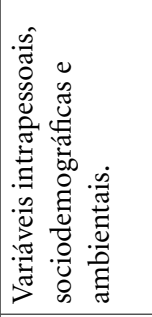 & 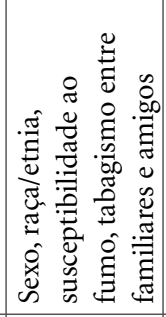 & 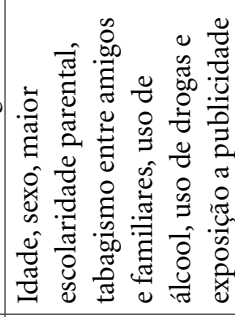 \\
\hline 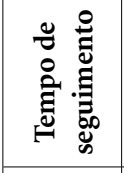 & 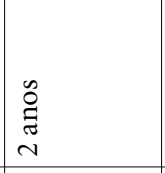 & 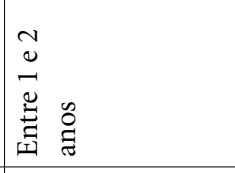 & 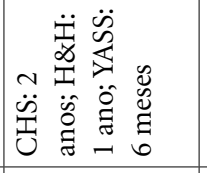 & 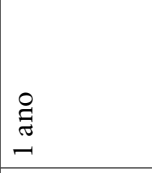 & 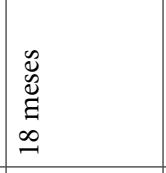 & 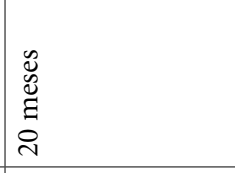 \\
\hline 象葛 & $\begin{array}{l}\infty \\
i n \\
i n\end{array}$ & $\frac{m}{2}$ & 今̂ & $\stackrel{m}{\stackrel{m}{*}}$ & $\hat{\hat{\sigma}}$ & in \\
\hline 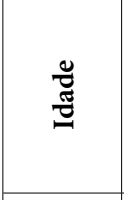 & 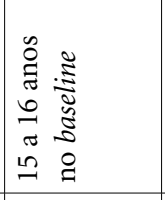 & 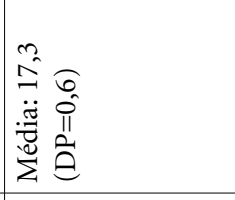 & 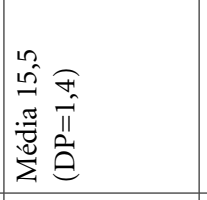 & 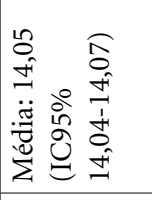 & 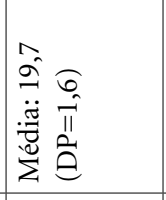 & 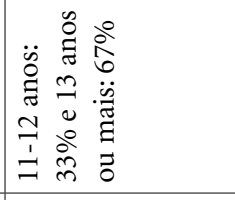 \\
\hline 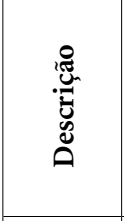 & 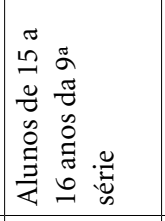 & 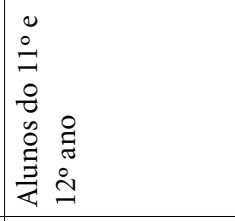 & 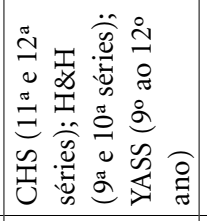 & 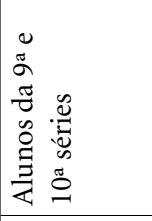 & 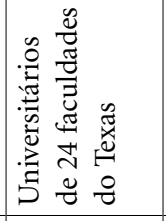 & 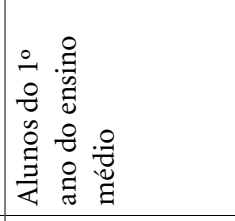 \\
\hline 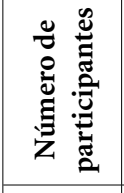 & 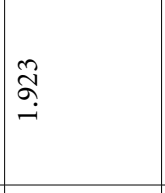 & 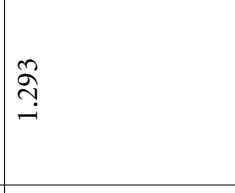 & $\begin{array}{l}\stackrel{0}{0} \\
\infty \\
\stackrel{+}{+}\end{array}$ & $\begin{array}{l}\stackrel{8}{8} \\
i n \\
i\end{array}$ & 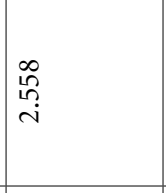 & $\begin{array}{l}\Omega \\
\hat{\sigma} \\
+ \\
+\end{array}$ \\
\hline 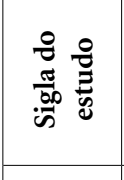 & 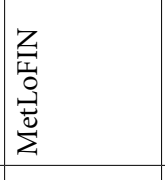 & 売 & 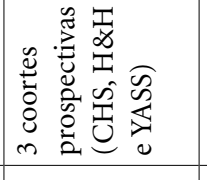 & 之 & 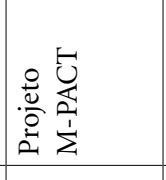 & $\bar{z}$ \\
\hline$\stackrel{0}{\tilde{a}}$ & 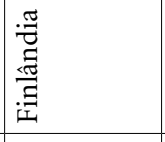 & 㟔 & 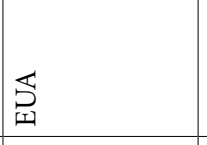 & 恿 & 岺 & 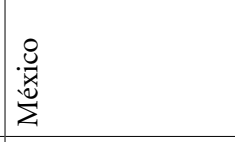 \\
\hline 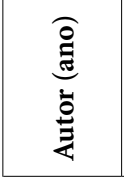 & 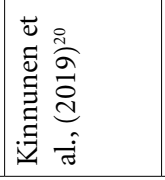 & 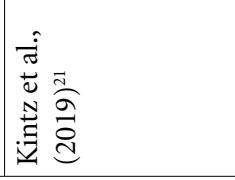 & 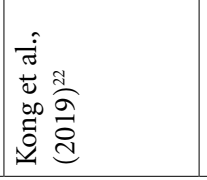 & 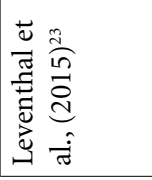 & 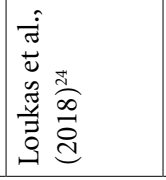 & 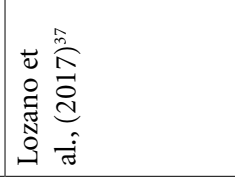 \\
\hline
\end{tabular}




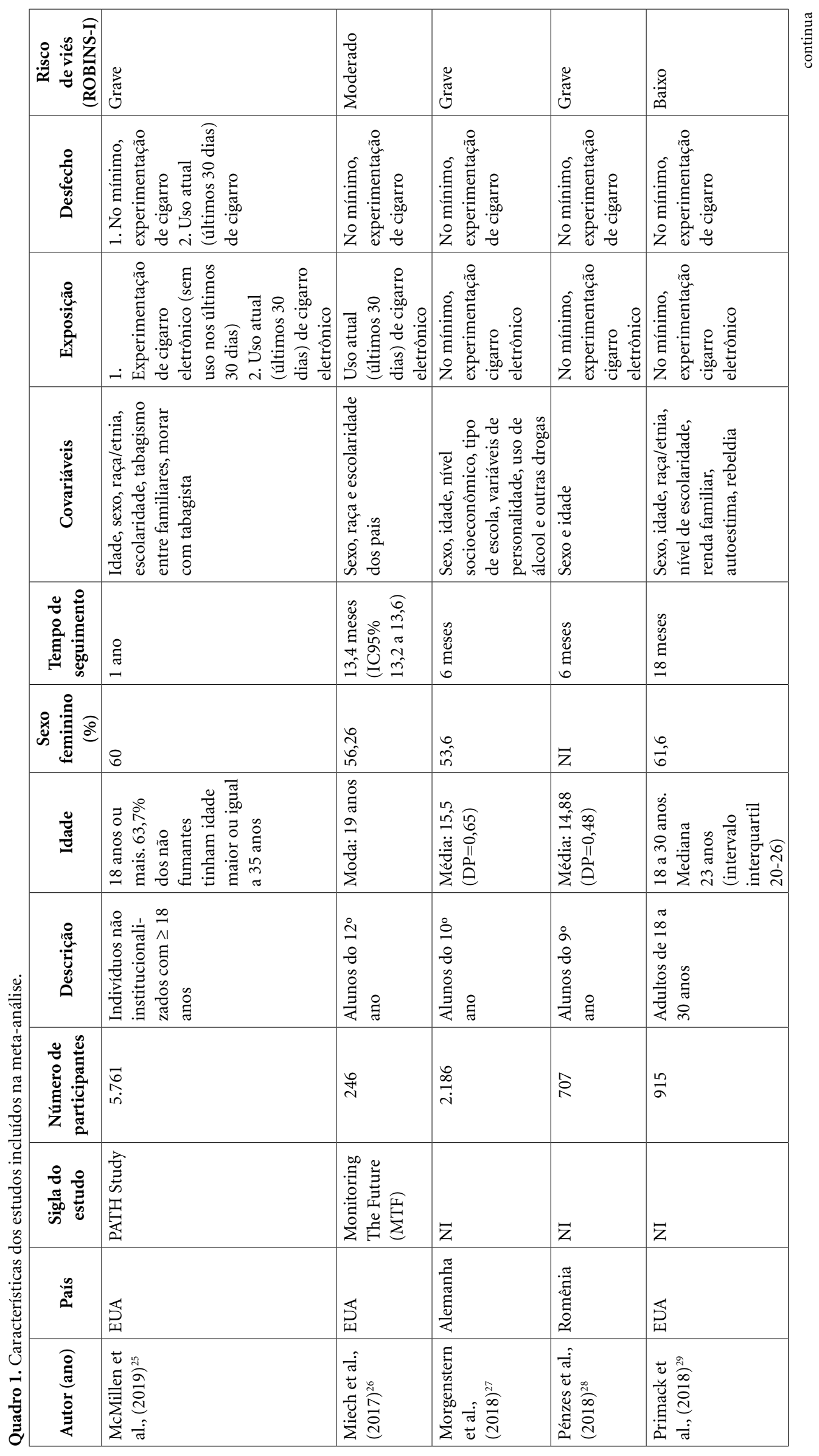




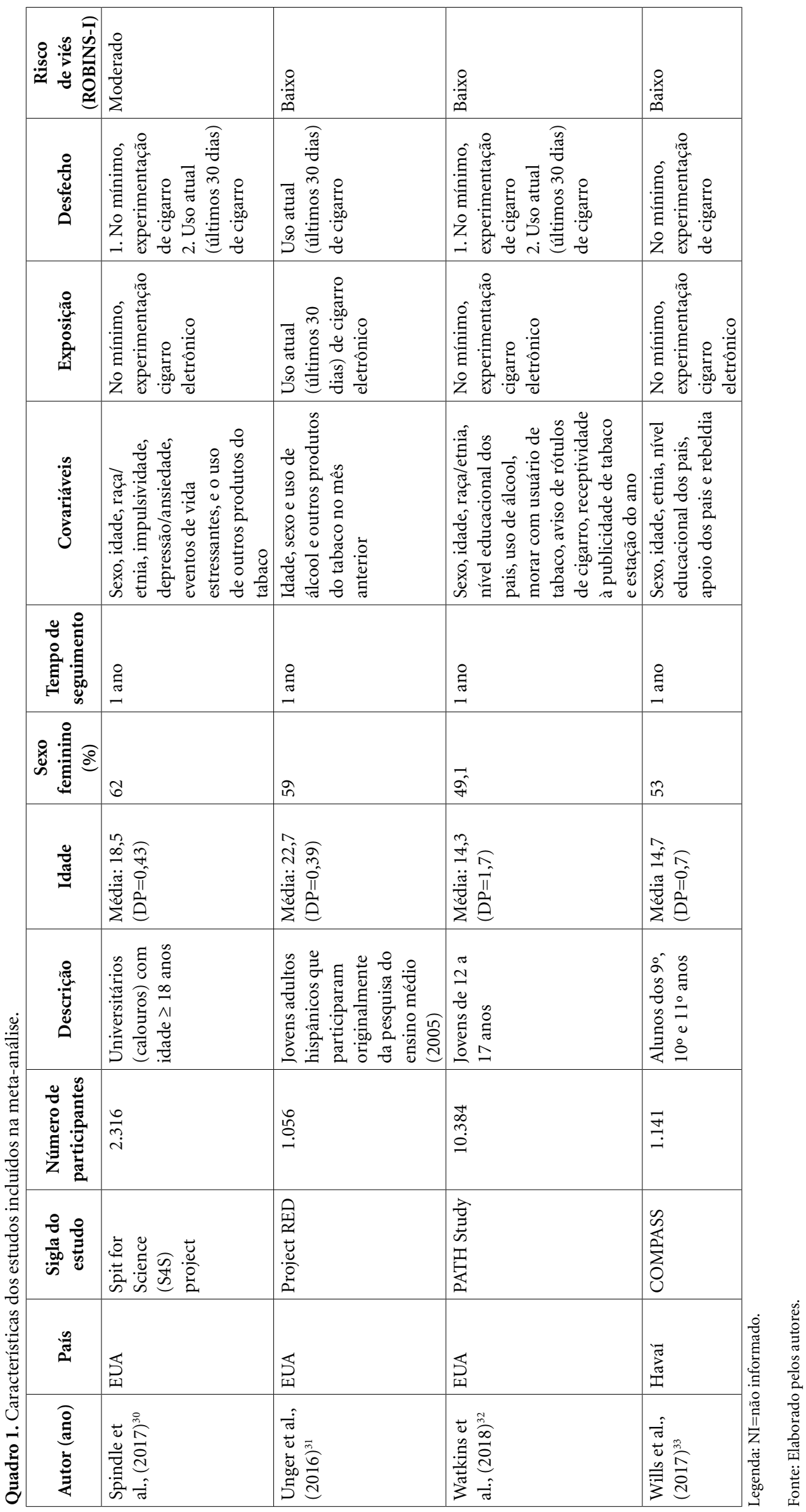




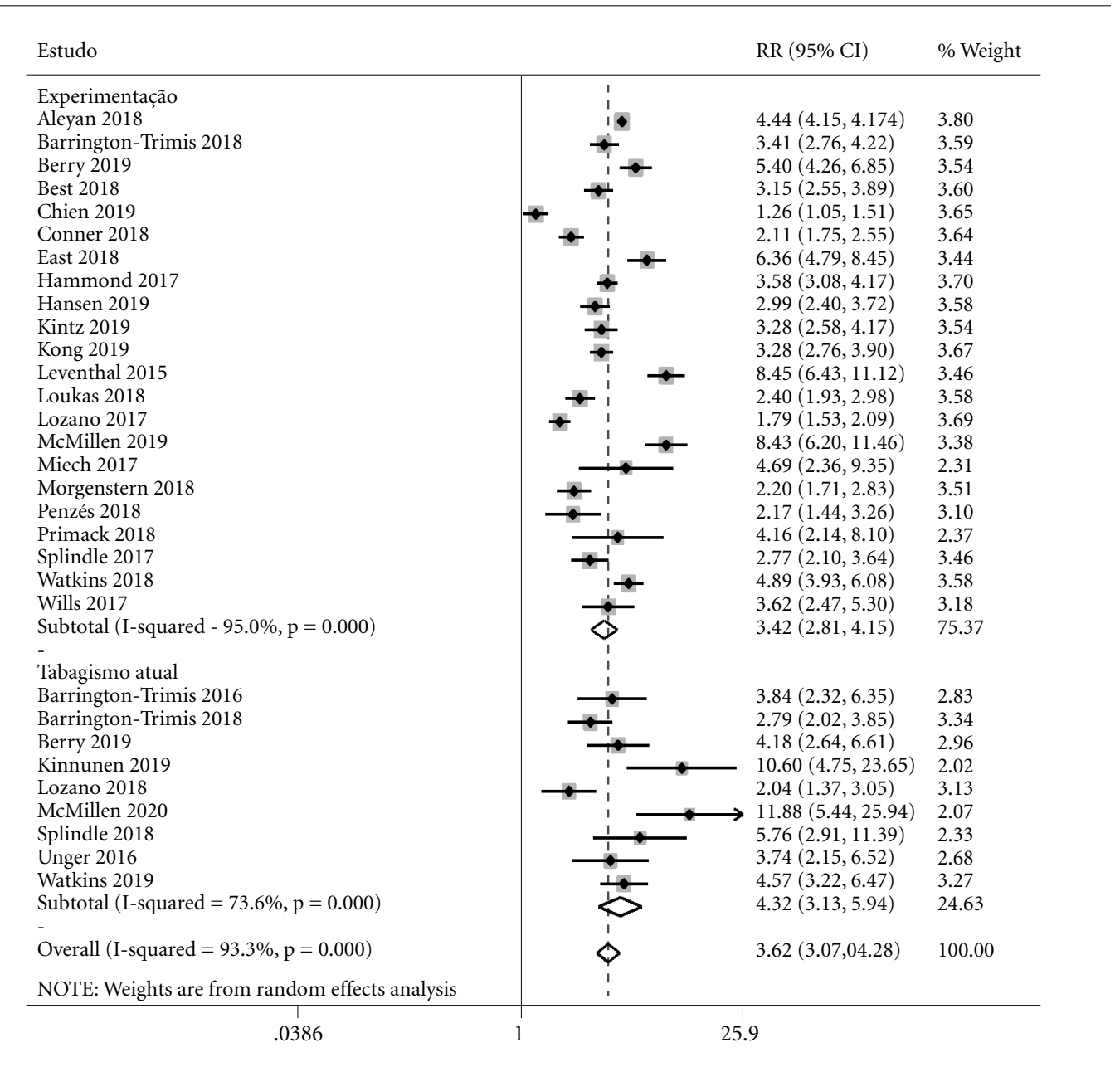

Figura 2. Meta-análise de iniciação do tabagismo (experimentação e uso atual) associada ao uso de cigarro eletrônico pelo modelo de Mantel-Haenszel de efeitos aleatórios.

Fonte: Elaborado pelos autores.

$\mathrm{Na}$ análise de subgrupo para o desfecho de experimentação, não se observou diferença significativa entre os grupos. Nessas análises, não se observa impacto dos subgrupos na heterogeneidade entre os estudos, já que a heterogeneidade se manteve significativa (Tabela 1 ).

Para o desfecho de uso atual de cigarros (últimos 30 dias), a análise de subgrupos também não aponta para diferença significativa entre as categorias. Nas três variáveis analisadas, houve uma redução na heterogeneidade entre os estudos que avaliaram população adulta, aqueles com tamanho amostral menor e com tempo de seguimento menor (Tabela 2).

Foi conduzida também uma meta-análise com as medidas de associação calculadas nos estudos primários, ou seja, ajustadas por co- variáveis. Nessa análise, a medida combinada para o desfecho de experimentação foi de 2,71 (IC95\% 2,28-3,14; $\mathrm{I}^{2}=83,3 \%$ ) e para o desfecho de tabagismo atual foi de 2,57 (IC95\% 1,72-3,42, $\left.\mathrm{I}^{2}=46,9 \%\right)$.

Com relação ao viés de publicação, o gráfico de funil (material suplementar) e o teste de Egger apontam para ausência desse tipo de viés $(\mathrm{t}=$ $0,89, \mathrm{p}$ valor $=0,379)$.

\section{Discussão}

Nesta revisão sistemática com meta-análise, os resultados dos estudos longitudinais foram consistentes, indicando que o uso de cigarros eletrônicos está associado ao risco aumentado de expe- 
Tabela 1. Análise de subgrupo para o desfecho de experimentação do tabagismo.

\begin{tabular}{lcccc}
\hline \multicolumn{1}{c}{ Grupos } & No de estudos & $\begin{array}{c}\text { Estimativa } \\
\text { combinada de risco } \\
\text { relativo (IC95\%) }\end{array}$ & $\mathbf{I}^{\mathbf{2}(\%)}$ & $\begin{array}{c}\text { Teste de } \\
\text { heterogeneidade } \\
\text { (p valor) }\end{array}$ \\
\hline $\begin{array}{l}\text { Idade na linha de base } \\
\quad \text { Até 18 anos }\end{array}$ & 17 & $3,29(2,65-4,09)$ & 95,7 & 0,000 \\
$\quad \begin{array}{l}\text { 18 anos ou mais } \\
\text { Tamanho amostral }\end{array}$ & 5 & $4,00(2,32-6,90)$ & 91,6 & 0,000 \\
$\quad$ Até 1.500 & 6 & $3,81(2,73-5,32)$ & 77,0 & 0,001 \\
$\quad$ 1.500 ou mais & 16 & $3,30(2,63-4,15)$ & 96,2 & 0,000 \\
Tempo de seguimento & 11 & $4,03(3,03-5,35)$ & 92,9 & 0,000 \\
$\quad$ Até um ano (inclusive) & 11 & $2,91(2,18-3,90)$ & 96,4 & 0,000 \\
$\quad$ Mais de um ano & & & & \\
\hline
\end{tabular}

Fonte: Elaborado pelos autores.

Tabela 2. Análise de subgrupo para o desfecho de tabagismo atual (últimos 30 dias).

\begin{tabular}{|c|c|c|c|c|}
\hline Grupos & No de estudos & $\begin{array}{c}\text { Estimativa } \\
\text { combinada de risco } \\
\text { relativo (IC95\%) }\end{array}$ & $I^{2}(\%)$ & $\begin{array}{c}\text { Teste de } \\
\text { heterogeneidade } \\
\text { ( } p \text { valor) }\end{array}$ \\
\hline \multicolumn{5}{|l|}{ Idade na linha de base } \\
\hline Até 18 anos & 6 & $3,76(2,64-5,33)$ & 74,1 & 0,002 \\
\hline 18 anos ou mais & 3 & $6,06(3,18-11,56)$ & 64,3 & 0,061 \\
\hline \multicolumn{5}{|l|}{ Tamanho amostral } \\
\hline Até 1.500 & 2 & $3,79(2,61-5,51)$ & 0,0 & 0,942 \\
\hline 1.500 ou mais & 7 & $4,58(3,03-6,91)$ & 80,3 & 0,000 \\
\hline \multicolumn{5}{|l|}{ Tempo de seguimento } \\
\hline Até um ano (inclusive) & 4 & $5,38(3,59-8,06)$ & 51,5 & 0,103 \\
\hline Mais de um ano & 5 & $3,61(2,39-5,46)$ & 73,6 & 0,000 \\
\hline
\end{tabular}

Fonte: Elaborado pelos autores.

rimentação e uso atual de cigarros convencionais. Os resultados apontando para essa associação foram encontrados tanto nas análises com os dados brutos, quanto na análise com as medidas de associação ajustadas.

Ainda que importante heterogeneidade tenha sido demonstrada para a quantificação dessa associação, todos os estudos apontam unidirecionalmente para o aumento significativo de iniciação ao tabagismo, levando a um risco combinado três vezes maior para experimentação, e quatro vezes maior para uso atual do cigarro convencional. Além disso, o risco de iniciação ao tabagismo foi maior tanto em adultos quanto em menores de 18 anos que utilizaram cigarro eletrônico, sendo tal efeito persistente mesmo em estudos maiores e com seguimento mais longo.

As associações verificadas no presente estudo corroboram os resultados apresentados em uma revisão sistemática com meta-análise publicada anteriormente, porém com menor número de estudos $^{3}$. Os autores observaram maior chance de iniciação ao tabagismo entre os indivíduos que utilizaram cigarros eletrônicos, com valores semelhantes tanto para experimentação, quanto para o uso de cigarros nos últimos 30 dias. Os resultados de outra revisão sistemática (sem meta-análise) de estudos longitudinais com tempo de seguimento de seis meses a 2,5 anos, também sugerem associação entre o início do tabagismo e o uso de cigarros eletrônicos, entre os nunca fumantes ${ }^{4}$.

Os cigarros eletrônicos se apresentam em uma ampla variedade de produtos, mas todos consistem em quatro elementos principais: um cartucho ou reservatório, um elemento de aquecimento, uma bateria de íons de lítio e um bocal por onde é feita a inalação. $\mathrm{O}$ cartucho contém uma solução líquida contendo quantidades va- 
riáveis de nicotina, aromas e outros produtos químicos dissolvidos em solventes como propilenoglicol e/ou glicerina vegetal. Ao fornecer nicotina por aerossolização e não por fumaça de tabaco, os cigarros eletrônicos evitam muitos dos subprodutos da combustão tradicional e, portanto, produzem menos toxinas ${ }^{38}$.

No entanto, os cigarros eletrônicos contêm aditivos e solventes que podem formar compostos tóxicos e cancerígenos; e liberar nanopartículas de metais tóxicos do dispositivo e do líquido durante o aquecimento ${ }^{38-40}$. Alguns estudos demonstraram que os riscos à saúde relacionados à utilização de cigarros eletrônicos podem ir além do impacto neurológico da nicotina ou dos efeitos pulmonares devido à inalação ${ }^{41,42}$. Estudo recente demonstrou, por exemplo, que agentes cancerígenos sabidamente relacionados ao câncer de bexiga estão presentes na urina de usuários de cigarros eletrônicos ${ }^{43}$. Além disso, desde 2019, milhares de usuários ficaram gravemente doentes em uma epidemia de lesões pulmonares associadas ao uso de cigarros eletrônicos (EVALI), nos Estados Unidos. Pacientes com EVALI apresentam um conjunto de sintomas respiratórios, gastrointestinais e constitucionais. O surto desta$\mathrm{ca}$ a heterogeneidade dos constituintes do líquido do cigarro eletrônico e põe em dúvida a segurança a longo prazo do seu uso ${ }^{44}$.

Os cigarros eletrônicos foram introduzidos no mercado com a justificativa de apoiar a cessação do tabagismo, substituindo-os por dispositivos que imitam a forma e a sensação dos cigarros $^{45}$. Entretanto, sua popularidade aumentou também entre os não fumantes ${ }^{46-48}$, impulsionada por uma maior aceitação social, um gosto mais atraente e uma aparência contemporânea em relação aos cigarros combustíveis tradicionais ${ }^{49,50}$ desafiando, portanto, as perspectivas atuais sobre sua segurança e eficácia como uma intervenção em saúde pública ${ }^{51}$.

Ainda nesse sentido, a sua associação com a iniciação do cigarro convencional, como demonstrada nesta revisão sistemática, potencializa ainda mais o impacto negativo do uso de cigarro eletrônico pelo consequente aumento da prevalência de tabagismo entre jovens, atualmente em declínio no Brasil ${ }^{5}$.

A transição de usuário de cigarro eletrônico para usuário de cigarro convencional pode ser explicada, entre outros, por aspectos comporta- mentais e fisiológicos, já que a utilização deste produto imita os comportamentos do tabagismo, como os movimentos mão-boca, inalação e expiração ${ }^{52}$. Além disso, adolescentes e adultos jovens que usam cigarros eletrônicos contendo nicotina podem se tornar dependentes dessa substância e como resultado, estar mais propensos a experimentar cigarros combustíveis e outras formas de nicotina inalável ${ }^{53}$. Por fim, o custo de manutenção do uso regular de cigarro eletrônico pode ser mais elevado do que o do cigarro convencional e isso contribuiria para essa transição, principalmente em indivíduos de classe econômica menos favorecida.

Esta revisão sistemática apresenta algumas limitações. A estratégia de busca foi semelhante, mas não a mesma, das revisões sistemáticas anteriores. Além disso, foi utilizado um filtro de data, podendo ocasionar perda de referências. Cinco estudos selecionados foram avaliados com risco de viés grave, com perdas significativas no seguimento em alguns deles. Em relação ao resultado de risco ajustado, diferentes covariáveis foram utilizadas na análise pelos estudos, considerando as especificidades das populações quanto a características de comportamento tabágico e seus fatores associados. Entretanto, a maioria dos estudos fez o ajuste, minimamente, por sexo, idade, alguma variável socioeconômica e exposição ao tabagismo dos pais, e, para esta revisão, realizamos preferencialmente a meta-análise com os dados brutos extraídos dos estudos.

Como pontos favoráveis é possível destacar que essa revisão sistemática incluiu grande número de estudos e de participantes. Os autores optaram por incluir somente estudos longitudinais com indivíduos não fumantes na linha de base. Portanto, há convicção de que a exposição ocorreu antes do desfecho garantindo a temporalidade da associação.

Assim, apesar da heterogeneidade estatística observada entre os estudos, tanto os resultados brutos como os ajustados são consistentes com o maior risco de iniciação ao tabagismo nos usuários de cigarro eletrônico. Portanto, as atuais evidências demonstradas neste estudo apontam para a ameaça que a liberação da comercialização de cigarros eletrônicos representa para o Brasil, principalmente trazendo como consequência importante o aumento na prevalência de tabagistas e suas doenças associadas. 


\section{Colaboradores}

LA Barufaldi, RL Guerra e RCR Albuquerque participaram da concepção e planejamento do trabalho, interpretação das evidências e redação do manuscrito. A Nascimento participou da concepção e planejamento do trabalho, interpretação das evidências e revisão crítica das versões preliminares e definitiva. RD Chança, MC Souza e LM Almeida participaram da concepção e planejamento do trabalho e revisão crítica das versões preliminares e definitiva. Todos os autores aprovaram a versão final do manuscrito.

\section{Referências}

1. National Institute on Drug Abuse. Vaping Devices (Electronic Cigarettes) [Internet]. 2020 [acessado 2019 mar 18]. Disponível em: www.drugabuse.gov/publications/drugfacts/vaping-devices-electronic-cigarettes

2. Hess CA, Olmedo P, Navas-Acien A, Goessler W, Cohen JE, Rule AM. E-cigarettes as a source of toxic and potentially carcinogenic metals. Environ Res 2017; 152:221-225.

3. Soneji S, Barrington-Trimis JL, Wills TA, Leventhal AM, Unger JB, Gibson LA, Yang JW, Primack BA, Andrews JA, Miech RA, Spindle TR, Dick DM, Eissenberg T, Hornik RC, Dang R, Sargent JD. Association between initial use of e-cigarettes and subsequent cigarette smoking among adolescents and young adults a systematic review and meta-analysis. JAMA Pediatr 2017; 171(8):788-797.

4. Glasser A, Abudayyeh H, Cantrell J, Niaura R. Patterns of E-Cigarette Use among Youth and Young Adults: Review of the Impact of E-Cigarettes on Cigarette Smoking. Nicotine Tob Res 2019; 21(10):1320-1330.

5. Oliveira-Campos M, Oliveira MM, Silva SU, Santos MAS, Barufaldi LA, Oliveira PPV, Andrade SCA, Andreazzi MAR, Moura L, Malta DC, Souza MFM. Risk and protection factors for chronic noncommunicable diseases in adolescents in Brazilian capitals. Rev Bras Epidemiol 2018; 21(Supl. 1):e180002.

6. Brasil. Ministério da Saúde (MS). Pesquisa nacional de saúde do escolar: 2015. Rio de Janeiro: IBGE; 2016.

7. Cavalcante TM, Szklo AS, Perez CA, Thrasher JF Szklo M, Ouimet J, Gravely S, Fong GT, Almeida LM. Conhecimento e uso de cigarros eletrônicos e percepção de risco no Brasil: Resultados de um país com requisitos regulatórios rígidos. Cad Saude Publica 2017; 33(Supl. 3):e00074416.

8. Bertoni N, Szklo A, De Boni R, Coutinho C, Vasconcellos M, Silva PN, Almeida LM, Bastos FI. Electronic cigarettes and narghile users in Brazil: Do they differ from cigarettes smokers? Addict Behav 2019; 98:106007.

9. Moher D, Liberati A, Tetzlaff J, Altman DG, PRISMA Group. Preferred reporting items for systematic reviews and meta-analyses: The PRISMA statement. PLoS Med 2009; 6(7):e1000097.

10. Ouzzani M, Hammady H, Fedorowicz Z, Elmagarmid A. Rayyan - a web and mobile app for systematic reviews [Internet]. Systematic Reviews; 2016 [acessado 2019 mar 18]. Disponível em: https://rayyan.qcri.org/ welcome.

11. Sterne JA, Hernán MA, Reeves BC, Savovi J, Berkman ND, Viswanathan M, Henry D, Altman DG, Ansari MT, Boutron I, Carpenter JR, Chan A-W, Churchill R, Deeks JJ, Hróbjartsson A, Kirkham J, Jüni P, Loke YK, Pigott TD, Ramsay CR, Regidor D, Rothstein HR, Sandhu L, Santaguida PL, Schünemann HJ, Shea B, Shrier I, Tugwell P, Turner L, Valentine JC, Waddington $\mathrm{H}$, Waters E, Wells GA, Whiting PF, Higgins JP. ROBINS -I: A tool for assessing risk of bias in non-randomised studies of interventions. BMJ 2016; 355:i4919.

12. Higgins JPT, Thompson SG. Quantifying heterogeneity in a meta-analysis. Stat Med 2002; 21(11):15391558 . 
13. Barrington-Trimis JL, Urman R, Berhane $\mathrm{K}$, Unger JB, Cruz TB, Pentz MA, Samet JM, Leventhal AM, McConnell R. E-cigarettes and future cigarette use. $\mathrm{Pe}$ diatrics 2016; 138(1):e20160379.

14. Barrington-Trimis JL, Kong G, Leventhal AM, Liu F, Mayer M, Cruz TB, Krishnan-Sarin S, McConnell R. E-cigarette Use and Subsequent Smoking Frequency Among Adolescents. Pediatrics 2018; 142(6): e20180486.

15. Berry KM, Fetterman JL, Benjamin EJ, Bhatnagar A, Barrington-Trimis JL, Leventhal AM, Stokes A. Association of Electronic Cigarette Use With Subsequent Initiation of Tobacco Cigarettes in US Youths. JAMA Netw Open 2019; 2(2):e187794.

16. Best C, Haseen F, Currie D, Ozakinci G, MacKintosh AM, Stead M, Eadie D, MacGregor A, Pearce J, Amos A, Frank J, Haw S. Relationship between trying an electronic cigarette and subsequent cigarette experimentation in Scottish adolescents: A cohort study. Tob Control 2018; 27(4):373-378.

17. Conner M, Grogan S, Simms-Ellis R, Flett K, SykesMuskett B, Cowap L, Lawton R, Armitage CJ, Meads D, Torgerson C, West R, Siddiqi K. Do electronic cigarettes increase cigarette smoking in UK adolescents? Evidence from a 12-month prospective study. Tob Control 2018; 27(4):365-372.

18. East K, Hitchman SC, Bakolis I, Williams S, Cheeseman H, Arnott D, McNeill A. The Association Between Smoking and Electronic Cigarette Use in a Cohort of Young People. J Adolesc Heal 2018; 62(5):539-547.

19. Hansen J, Janssen J, Morgenstern M, Hanewinkel PD. E-Cigarette Use and Later Use of Conventional Cigarettes: Results of a Prospective Observational Study over 2 Years. Pneumologie 2020; 74(1):39-45.

20. Kinnunen JM, Ollila H, Minkkinen J, Lindfors PL, Timberlake DS, Rimpelä AH. Nicotine matters in predicting subsequent smoking after e-cigarette experimentation: A longitudinal study among Finnish adolescents. Drug Alcohol Depend 2019; 201:182-187.

21. Kintz N, Liu M, Chou CP, Urman R, Berhane K, Unger JB, Cruz TB, McConnell R, Barrington-Trimis JL. Risk factors associated with subsequent initiation of cigarettes and e-cigarettes in adolescence: A structural equation modeling approach. Drug Alcohol Depend 2020; 207:107676.

22. Kong G, Mayer ME, Barrington-Trimis JL, McConnell R, Leventhal AM, Krishnan-Sarin S. Longitudinal associations between use and co-use of cigars and cigarettes: A pooled analysis of three adolescent cohorts. Drug Alcohol Depend 2019; 201:45-48.

23. Leventhal AM, Strong DR, Kirkpatrick MG, Unger JB, Sussman S, Riggs NR, Stone MD, Khoddam R, Samet JM, Audrain-McGovern J. Association of electronic cigarette use with initiation of combustible tobacco product smoking in early adolescence. JAMA 2015; 314(7):700-707.

24. Loukas A, Marti CN, Cooper M, Pasch KE, Perry CL. Exclusive e-cigarette use predicts cigarette initiation among college students. Addict Behav 2018; 76:343347.
25. McMillen R, Klein JD, Wilson K, Winickoff JP, Tanski S. E-Cigarette Use and Future Cigarette Initiation Among Never Smokers and Relapse Among Former Smokers in the PATH Study. Public Health Rep 2019; 134(5):528-536.

26. Miech R, Patrick ME, O’Malley PM, Johnston LD. E-cigarette use as a predictor of cigarette smoking: results from a 1-year follow-up of a national sample of 12th grade students. Tob Control 2017; 26(e2):e106-e111.

27. Morgenstern M, Nies A, Goecke M, Hanewinkel R. Ecigarettes and the use of conventional cigarettes - A cohort study in 10th grade students in Germany. Dtsch Arztebl Int 2018; 115(14):243-248.

28. Pénzes M, Foley KL, Nădășan V, Paulik E, Ábrám Z, Urbán R. Bidirectional associations of e-cigarette, conventional cigarette and waterpipe experimentation among adolescents: A cross-lagged model. Addict Behav 2018; 80:59-64.

29. Primack BA, Shensa A, Sidani JE, Hoffman BL, Soneji S, Sargent JD, Hoffman RM, Fine MJ. Initiation of Traditional Cigarette Smoking after Electronic Cigarette Use Among Tobacco-Naïve US Young Adults. Am J Med 2018; 131(4):443.e1-443.e9.

30. Spindle TR, Hiler MM, Cooke ME, Eissenberg T, Kendler KS, Dick DM. Electronic cigarette use and uptake of cigarette smoking: A longitudinal examination of U.S. college students. Addict Behav 2017; 67:66-72.

31. Unger JB, Soto DW, Leventhal A. E-cigarette use and subsequent cigarette and marijuana use among Hispanic young adults. Drug Alcohol Depend 2016; 163:261-264.

32. Lea Watkins S, Glantz SA, Chaffee BW. Association of noncigarette tobacco product use with future cigarette smoking among youth in the population assessment of tobacco and health (PATH) study, 2013-2015. JAMA Pediatr 2018; 172(2):181-187.

33. Wills TA, Knight R, Sargent JD, Gibbons FX, Pagano I, Williams RJ. Longitudinal study of e-cigarette use and onset of cigarette smoking among high school students in Hawaii. Tob Control 2017; 26(1):34-39.

34. Aleyan S, Cole A, Qian W, Leatherdale ST. Risky business: A longitudinal study examining cigarette smoking initiation among susceptible and non-susceptible e-cigarette users in Canada. BMJ Open 2018; 8(5):e021080.

35. Hammond D, Reid JL, Cole AG, Leatherdale ST. Electronic cigarette use and smoking initiation among youth: A longitudinal cohort study. CMAJ 2017; 189(43):E1328-E1336.

36. Chien YN, Gao W, Sanna M, Chen PL, Chen YH, Glantz S, Chiou H-Y. Electronic cigarette use and smoking initiation in Taiwan: Evidence from the first prospective study in Asia. Int J Environ Res Public Health 2019; 16(7):1145.

37. Lozano P, Barrientos-Gutierrez I, Arillo-Santillan E, Morello P, Mejia R, Sargent JD, Thrasher JF. A longitudinal study of electronic cigarette use and onset of conventional cigarette smoking and marijuana use among Mexican adolescents. Drug Alcohol Depend 2017; 180:427-430. 
38. Goniewicz ML, Knysak J, Gawron M, Kosmider L Sobczak A, Kurek J, Prokopowicz A, Jablonska-Czapla M, Rosik-Dulewska C, Havel C, Jacob 3rd P, Benowitz $\mathrm{N}$. Levels of selected carcinogens and toxicants in vapour from electronic cigarettes. Tob Control 2014; 23(2):133-139.

39. Mcrobbie H, Phillips A, Goniewicz ML, Smith KM, Knight-west O, Przulj D, Hajek P. Effects of Switching to Electronic Cigarettes with and without Concurrent Smoking on Exposure to Nicotine, Carbon Monoxide, and Acrolein. Cancer Prev Res (Phila) 2015; 8(9):873878.

40. Connell GO, Graff DW, Ruiz CDD. Reductions in biomarkers of exposure ( $\mathrm{BoE}$ ) to harmful or potentially harmful constituents (HPHCs) following partial or complete substitution of cigarettes with electronic cigarettes in adult smokers. Toxicol Mech Methods 2016; 26(6):443-454.

41. Butt YM, Smith ML, Tazelaar HD, Vaszar LT, Swanson KL, Cecchini MJ, Boland JM, Bois MC, Boyum JH, Froemming AT, Khoor A, Mira-Avendano I, Patel A, Larsen BT. Pathology of Vaping-Associated Lung Injury. N Engl J Med 2019; 381(18):1780-1781.

42. Fuller TW, Acharya AP, Meyyappan T, Yu M, Bhaskar G, Little SR, Tarin TV. Comparison of Bladder Carcinogens in the Urine of E-cigarette Users Versus Non E-cigarette Using Controls. Sci Rep 2018; 8(1):507.

43. Bjurlin MA, Matulewicz RS, Roberts TR, Dearing BA Schatz D, Sherman S, Gordon T, El Shahawy O. Carcinogen Biomarkers in the Urine of Electronic Cigarette Users and Implications for the Development of Bladder Cancer: A Systematic Review. Eur Urol Oncol 2020; S2588-9311(20):30029-30028.

44. Cao DJ, Aldy K, Hsu S, McGetrick M, Verbeck G, Silva I, Feng S-Y. Review of Health Consequences of Electronic Cigarettes and the Outbreak of Electronic Cigarette, or Vaping, Product Use-Associated Lung Injury. J Med Toxicol 2020; 16(3):295-310.

45. Siegel MB, Tanwar KL, Wood KS. Electronic Cigarettes As a Smoking-Cessation Tool. AMEPRE 2011; 40(4):472-475.

46. Mirbolouk M, Charkhchi P, Kianoush S, Uddin SMI, Orimoloye OA, Jaber R, Bhatnagar A, Benjamin EJ, Hall ME, DeFilippis AP, Maziak W, Nasir K, Blaha MJ. Prevalence and Distribution of E-Cigarette Use Among U.S. Adults: Behavioral Risk Factor Surveillance System, 2016 Mohammadhassan. Ann Intern Med 2018; 169(7):429-438.

47. Shiplo S, Czoli CD, Hammond D. E-cigarette use in Canada: prevalence and patterns of use in a regulated market. BMJ Open 2015; 5(8):e007971.

48. Filippidis FT, Laverty AA, Gerovasili V, Vardavas CI. Two-year trends and predictors of e-cigarette use in 27 European Union member states. Tob Control 2017; 26(1):98-104.

49. Rubinstein ML, Delucchi K, Benowitz NL, Ramo DE. Adolescent Exposure to Toxic Volatile Organic Chemicals From E-Cigarettes. Pediatrics 2020; 141(4):e20173557.
50. Kasza KA, Ambrose BK, Ph D, Conway KP, Ph D, Borek N, Taylor K, Goniewicz ML, Cummings KM, Sharma E, Pearson JL, Green VR, Kaufman AR, Bansal-Travers M, Travers MJ, Kwan J, Tworek C, Cheng Y-C, Yang L, Pharris-Ciurej N, Bemmel DM, Backinger CL, Compton WM, Hyland AJ. Tobacco-Product Use by Adults and Youths in the United States in 2013 and 2014. N Engl J Med 2017; 376(4):342-353.

51. Amin s, Dunn AG, Laranjo L. Social Influence in the Uptake and Use of Electronic Cigarettes: A Systematic Review. Am J Prev Med 2020; 58(1):129-141.

52. Primack BA, Soneji S, Stoolmiller M, Fine MJ, Sargent JD. Progression to traditional cigarette smoking after electronic cigarette use among us adolescents and young adults. JAMA Pediatr 2015; 169(11):1018-1023.

53. Goel R, Durand E, Trushin N, Prokopczyk B, Foulds J. Highly Reactive Free Radicals in Electronic Cigarette Aerosols Reema. Chem Res Toxicol 2016; 28(9):16751677.

Artigo apresentado em 24/06/2020

Aprovado em 18/10/2020

Versão final apresentada em 20/10/2020

Editores-chefes: Romeu Gomes, Antônio Augusto Moura da Silva 
